\title{
Analysis of Low Voltage Cable Insulation Detection Technology
}

\author{
Dandan $\mathrm{Su}^{1, \mathrm{a}}$
}

${ }^{1}$ Wuxi Institute of Arts \& Technology, Yixing, Jiangsu, 214206

\author{
Keywords: Cable Insulation, Detection Technology, Low Voltage
}

\begin{abstract}
In this paper, the mechanism of aging of insulating materials, the causes of insulation leakage current and the related circuit models are described. On this basis, the principle and advantages and disadvantages of various insulation detection technologies for wire and cable are analyzed. Secondly, based on the actual demand of low voltage distribution network insulation detection, this paper develops a digital megger based on single chip microcomputer. The working principle and performance of the paper are described in detail from the aspects of hardware and software. In addition, through the establishment of the corresponding circuit model, the paper discusses the feasibility and superiority of superposition method on the online insulation detection technology of IT low voltage distribution network, and obtains the preliminary verification by Matlab software simulation.
\end{abstract}

\section{Introduction}

Insulation damage is complicated and varied. Electrical equipment in the manufacture, transportation, installation and operation of the process may have insulation damage and deterioration and this damage and degradation is mainly due to mechanical effects, thermal effects, electric field, chemical corrosion and other factors combined effect produced. Electrical equipment insulation defects are mainly two categories: one is a concentrated defect, refers to focus on a part of the insulation or a few parts of the more serious defects. It can be divided into penetrating defects and non-penetrating defects. The potential for such defects is the fastest, and the likelihood of serious consequences is greater. But the concentration of defects is more obvious, and therefore easier to detect in advance. The other is the distribution of defects, that the overall insulation performance. It is a universal insulation deterioration that is slowly evolving and developing. Such defects are more difficult to attract enough attention, often from the distribution of defects evolved to the concentration of defects, until the consequences were taken seriously. Early detection of insulation distribution defects on the insulation detection technology put forward higher requirements. Insulation properties of insulating materials in the use of the process of deterioration, some are reversible, and some are irreversible. Reversible deterioration such as moisture, etc., after drying and other targeted treatment can be restored to the original characteristics; and irreversible deterioration is caused by other causes of insulation properties cannot be restored, these changes are known as aging.

There are many different features that characterize the degree of deterioration of different insulation systems. Insulation characteristics can be divided into two categories: the direct characterization of the residual life of the insulation characteristics, such as electrical strength, mechanical strength, etc; indirect characterization of the residual life of insulation characteristics, including insulation resistance, dielectric loss tangent, leakage Current, partial discharge, oil in the gas content, the oil slightly water content. The purpose of insulation testing is to detect these features, and accordingly determine the insulation conditions are good or bad, in advance to do the equipment maintenance and replacement. The direct feature quantity is obtained by a destructive test method, such as a high pressure withstand voltage test, and the indirect feature quantity can be obtained by a non-destructive test method. In addition, some new feature factors such as the second current surge point, the DC component, the UHF discharge spectrum, the ultrasonic vibration characteristics, etc., have been studied on the law of the different aging factors such as electricity, heat and water. The Insulation aging is a function of time and aging factors, the degree of insulation aging according to its performance changes to determine. The power equipment in operation 
requires periodic or on-line inspection to monitor changes in its insulation performance, replace insulation or damage to the insulation before it reaches the threshold, to ensure proper operation of the electrical equipment without causing an accident.

\section{Insulation Diagnosis Technology for Cables}

Measuring the insulation resistance of the instrument is called the megger, the traditional hand shake to produce the voltage of the shake table, with the development of digital technology, there have been a variety of excellent performance megger.

The traditional conventional stop operation of the insulation test interval is long, it is not easy to find equipment insulation defects in time, and the test will cause some economic losses due to power outages. With the development of detection technology, the continuous development of computer technology and the increasing importance of power equipment safety, online insulation diagnosis technology has been paid more and more attention. Online detection and stop running detection compared to the main advantages such as several advantages:

In the system work to complete the measurement, no power failure, improve the power supply efficiency.

Because it is measured at the actual operating voltage, it is more realistic than the measurement at the time of stopping the operation to reflect the insulation of the power equipment in the operating state.

On-line insulation monitoring device can be measured at any time on the system insulation conditions, more timely detection of insulation defects and failures, to avoid the blindness of the traditional preventive test.

It is helpful to establish the database of insulation of electric power equipment and analyze the trend of insulation deterioration based on the database of historical measurement records, forecast the residual insulation life, and pre-detect the potential faults early.

On-line monitoring helps to achieve centralized monitoring and computer automation monitoring, is conducive to the realization of insulation monitoring information and insulation diagnosis of intelligent.

Because it is not under test high pressure test, will not damage the electrical equipment insulation performance.

No temporary wiring is required for each measurement, which reduces the labor intensity of the tester, improves test efficiency and reduces test costs. Thus, insulation on-line monitoring for the safety of the grid, economic and efficient operation is of great significance. However, to achieve on-line monitoring, the following key issues need to be addressed:

There are a lot of noise and harmonics in the condition of grid operation. On-line monitoring is faced with the technical problems of extracting and accurately reducing the weak characteristic signals from these complicated noise and harmonics.

Because the on-line test power signal is different from the power frequency AC signal of the grid, it is one of the problems in the online measurement to choose what kind of test signal generator to reduce the influence of the test signal on the normal operation of the grid load.

On-line monitoring In addition to accurately picking out the effective characteristic signals from noise, it is also important to consider how to effectively superimpose the test source on the grid and minimize the impact of the ground line on the grid in the event of a grid failure (eg short circuit).

\section{Cable Insulation Online Diagnostics}

The traditional conventional stop operation of the insulation test interval is long, it is not easy to find equipment insulation defects in time, and the test will cause some economic losses due to power outages. With the development of detection technology, the continuous development of computer technology and the increasing importance of power equipment safety, online insulation diagnosis technology has been paid more and more attention. Online detection and stop running detection compared to the main advantages such as several advantages: 
In the system work to complete the measurement, no power failure, improve the power supply efficiency.

Because it is measured under the actual operating voltage, it is more realistic than the measurement at the time of stopping the operation to reflect the insulation condition of the power equipment in the running state.

On-line insulation monitoring device can be measured at any time on the system insulation, can be more timely detection of insulation defects and failures, to avoid the blindness of the traditional preventive test.

It is helpful to establish the database of insulation of electric power equipment and analyze the trend of insulation deterioration based on the database of historical measurement records, forecast the residual insulation life, and pre-detect the potential faults early.

On-line monitoring helps to achieve centralized monitoring and computer automation monitoring, is conducive to the realization of insulation monitoring information and insulation diagnosis of intelligent.

Failure to test under high pressure tests will not damage the insulation performance of electrical equipment.

No need for each time to set the temporary wiring wiring, reducing the labor intensity of the test staff to improve the test efficiency and reduce test costs. Thus, insulation on-line monitoring for the safety of the grid, economic and efficient operation is of great significance. However, to achieve on-line monitoring, the following key issues need to be addressed:

There are a lot of noise and harmonics in the condition of grid operation. On-line monitoring is faced with the technical problems of extracting and accurately reducing the weak characteristic signals from these complicated noise and harmonics.

Because the online test power signal is different from the power frequency AC power signal, so choose what kind of test signal generator to reduce the test signal on the normal operation of the power grid load is also one of the problems facing the online measurement.

On-line monitoring In addition to accurately picking out the effective characteristic signals from noise, consider how to effectively superimpose the test source on the grid and minimize the impact of the ground line on the grid in the event of a grid failure (eg short circuit) The The main methods are DC superposition method, DC component method, on-line $\tan \delta$ method, low frequency superposition method, AC superposition method and so on.

\section{Low Voltage Distribution Network Insulation on-Line Detection}

According to IEC364-3 standard, low-voltage distribution network can be divided into three forms: IT, TN, TT, TN distribution network which can be divided into TN-C, TN-S-1, TN-S-2, TN-CS , TN-C-TT and other sub-forms. The name of the law is mainly based on the system grounding method: the first letter that the low-voltage system to ground relations, $\mathrm{T}$ that a little ground, or by a small impedance ground, I said all live parts and earth insulation or artificial neutral ground; The second letter indicates that the electrically conductive part of the electrical device is exposed to ground, that is, $\mathrm{T}$ indicates that the exposed conductive part of the electrical device is directly electrically connected to the earth and is independent of any ground point in the low voltage system and $\mathrm{N}$ is the exposed conductive part of the electrical device And the low voltage system ground point has a direct electrical connection; the third letter that the system neutral line $\mathrm{N}$ and the protection line PEE combination, where S meaning $\mathrm{N}$ and PEE is completely separate, that is, by the two cables were born, $\mathrm{C}$ means that $\mathrm{N}$ is shared with PEE.

The insulation resistance of the cable is mainly affected by the size of the insulation resistance. For low-voltage distribution networks, the proportion of capacitive leakage current is not large, but once the line is relatively long, in some demanding occasions (such as hospitals, mines, etc.) capacitive leakage effect cannot be ignored. On the one hand, it will affect the accuracy of the insulation resistance measurement; the other hand, in some automatic tracking compensatory leakage protection system, the capacitive leakage current measurement accuracy requirements higher, otherwise, the performance of such systems It is difficult to improve. In addition, from the 
actual measurement results, the insulation DC resistance is much smaller than the AC resistance, when the use of DC superposition method measured data show that low-voltage distribution network insulation level within the safe range, and cannot completely rule out insecurity exist. Therefore, the use of AC superposition method on-line detection of insulation impedance as the basis for judging the insulation conditions than the use of DC superposition method measured DC insulation resistance closer to the actual situation, with a higher level of security. At the same time, the sampling alternating superposition method can isolate the capacitive component and the resistive component in the insulating leakage current to fully reproduce the actual insulation condition.

Overlay impedance can be selected for reactance, or resistance can be selected. Reactance will cause the current flow phase shift, and pure resistance will not have such an effect, at the same time, the use of resistance than the use of reactivity easier. The choice of Rs should take into account that it is also a load of three-phase alternating current, which consumes heat to produce heat, so it should not be too small; but if it is too large, the characteristic current will form a relatively large pressure drop on it, affecting the measurement. Take the total power of about $1.5 \mathrm{~W}$ is more appropriate value. That is, if the rms value of the distribution network phase is $220 \mathrm{~V}$, the value of Rs may be about $100 \mathrm{~K} \Omega$. Rm is the sampling resistor, its size is related to the range of insulation resistance can be measured, if too small, the voltage to the voltage is too small, to the subsequent processing inconvenience, if too large, the characteristic current will be too small , But also to the impact of measurement. Select $\mathrm{Rm}$ for the maximum range of 1/10000 more appropriate, for example, require a maximum range of $10 \mathrm{G} \Omega$, you can choose $\mathrm{Rm} 1 \mathrm{M} \Omega$, then if the test power amplitude of $20 \mathrm{~V}$, ignore the other impedance of the loop, $\mathrm{Rm}$ can be $2 \mathrm{mV}$ voltage amplitude , More suitable for follow-up circuit processing.

\section{Conclusion}

The low frequency superposition method is suitable for the on-line detection of low-voltage distribution network of neutral point ungrounded and can adapt to the capacitive leakage current of the grid to accurately extract the value of resistive leakage current. However, this method for the signal sampling and reduction accuracy requirements are relatively high, the need to use faster system implementation. Using high-speed AD, high-speed DSP as the core of the data acquisition system to FFT for the filter algorithm is more appropriate choice. Low-frequency superposition method in the practical application of whether to meet the requirements of the ground pressure distribution network insulation detection, IT-type distribution network in addition to the frequency of noise in the field, there are mainly what the nature of the noise and other engineering problems have yet to be explored in the follow-up practice.

\section{References}

[1] Luo Junhua, Zhou Zuchun, Zhang Li. Optimization of voltage amplitude and test time of VLF voltage test [J]. High Voltage Technology, 2006 (06)

[2] Wen Xishan, You Peng. Effects of water tree on XLPE cable insulation resistance and DC component [J]. Journal of Wuhan University, 2005 (06)

[3] Zhou Li. Insulation resistance measurement - absorption ratio test [J]. Journal of Shanghai Second Polytechnic University, 2004 (02)

[4] Luo Junhua, Yang Liming, Shi Jikang, Zhu Haigang. Power cable and test technology review [J]. High Voltage Technology, 2004 (11)

[5] Qian Qiuping. Study on Insulation Aging Diagnosis Technology of Cross-linked Polyethylene Cable [J]. Popular Science and Technology, 2004 (11) 